\title{
Correction: Experimental Study of In-vivo Dosimetry Using Glass Rod Dosimeters to Minimize the Initialization
}

\author{
[J. Korean Phys. Soc. 72, 628 (2018)] \\ DOI: $10.3938 / \mathrm{jkps} .72 .628$ \\ Hosang JeON, Jiho NAM, Jayoung LEE and Juhye LEE \\ Department of Radiation Oncology and Research Institute for Convergence of Biomedical Science and Technology, \\ Pusan National University Yangsan Hospital, Yangsan 50612, Korea \\ Dahl PARK,* Wontaek KIM, Yongkan KI and Donghyun KIM \\ Department of Radiation Oncology, Pusan National University Hospital, Busan 46241, Korea
}

DOI: $10.3938 / \mathrm{jkps} .75 .750$

The affiliation of Yongkan Ki should be corrected as follow:

Yongkan KI

Department of Radiation Oncology, Pusan National University School of Medicine, Busan 46241, Korea

*E-mail: dpark411@gmail.com 